\title{
REDUCTION THEOREMS FOR RELATIVE GROTHENDIECK RINGS $\left.{ }^{(}\right)$
}

\author{
BY \\ T. Y. LAM AND I. REINER
}

1. Introduction. Relative Grothendieck rings arise naturally when one considers modular representations of a finite group $G$, and their restrictions to some fixed subgroup $H$ of $G$. This article continues our earlier work on the subject [4], but can be read independently of that work.

Let $G$ be a finite group, and let $\Omega$ be a field of characteristic $p$, where we assume $p \neq 0$ to avoid trivial cases. By a " $G$-module" we mean always a finitely generated left $\Omega G$-module. Form the free abelian group $\mathscr{A}$ on the symbols $[M]$, where $M$ ranges over the isomorphism classes of $G$-modules; let $\mathscr{B}$ be the subgroup of $\mathscr{A}$ generated by all expressions $\left[M_{1}\right]-\left[M_{2}\right]-\left[M_{3}\right]$, where $M_{1} \cong M_{2} \oplus M_{3}$. The factor group $\mathscr{A} / \mathscr{B}$ will be called the Green ring or representation ring of $G$. We shall denote it by $a(G, G)$, in order to conform with notation to be introduced later. Define multiplication in $a(G, G)$ by

$$
[M][N]=\left[M \otimes_{\Omega} N\right],
$$

where each $g \in G$ acts on $M \otimes N$ according to the usual formula: $g(m \otimes n)$ $=g m \otimes g n$. Then $a(G, G)$ becomes a commutative ring whose identity element is $\left[1_{G}\right]$, where $1_{G}$ denotes the 1-representation of $G$

Now let

$$
E: 0 \rightarrow L \rightarrow M \rightarrow N \rightarrow 0
$$

be an exact sequence of $G$-modules, and define

$$
\gamma_{E}=[M]-[L]-[N] \in a(G, G) .
$$

Denote by $i(G, H)$ the additive subgroup of $a(G, G)$ generated by the expressions $\gamma_{E}$, where $E$ ranges over all exact sequences of $G$-modules which are split when restricted to $H$. Then $i(G, H)$ is an ideal in $a(G, G)$, and we define the relative Grothendieck ring $a(G, H)$ to be the factor $\operatorname{ring} a(G, H)=a(G, G) / i(G, H)$.

When $H=G$, the ideal $i(G, G)=0$, and in this case $a(G, H)$ coincides with the Green ring $a(G, G)$; this explains the notation $a(G, G)$ for the Green ring. Note that $a(G, G)$ is a free $Z$-module on the symbols $[M], M=$ indecomposable $G$ module.

Presented to the Society, August 21, 1968; received by the editors August 5, 1968.

( ${ }^{1}$ This research was supported by the National Science Foundation. 
On the other hand, when $H=1$, the relative Grothendieck ring $a(G, 1)$ is the $Z$-free module spanned by the irreducible $G$-modules. Thus $a(G, 1)$ is isomorphic to the ring of generalized Brauer characters of $G$. (See $[2, \S 82]$.)

There are surjective ring homomorphisms

$$
a(G, G) \rightarrow a(G, H), \quad a(G, H) \rightarrow a(G, 1) .
$$

Thus we expect that a relation $[M]=[N]$ in $a(G, H)$ will yield more information about the modules $M$ and $N$ than merely that they have the same Brauer characters (and the same composition factors). On the other hand, this relation may hold true even when $M, N$ are not isomorphic, so that $a(G, H)$ is easier to investigate than the Green ring $a(G, G)$. We hope that eventually we can use relative Grothendieck rings to study modular representations of groups with noncyclic Sylow p-subgroups.

Our previous work [4] dealt mainly with the structure of $a(G, H)$ when $H$ was a normal cyclic $p$-subgroup of $G$. In the present paper, we drop the restrictions on $H$, and obtain some "reduction formulas" which can be used in the calculation of $a(G, H)$. The principal results are Theorems 3.2 and 5.1.

2. Preliminary results. Throughout this paper, we assume that $G$ is a finite group, with subgroups $H, K$ satisfying $H \subset K \subset G$, where the inclusions need not be proper. In this section we shall give some miscellaneous propositions needed later.

The restriction map, taking $G$-modules into $K$-modules, induces a ring homomorphism

$$
a(G, H) \rightarrow a(K, H) .
$$

On the other hand, each $K$-module $M$ determines an induced $G$-module $M^{G}$, given by

$$
M^{G}=\Omega G \otimes_{\Omega K} M
$$

If

$$
E: 0 \longrightarrow L \stackrel{\lambda}{\longrightarrow} M \stackrel{\mu}{\longrightarrow} N \longrightarrow 0
$$

is an exact sequence of $K$-modules, there is an exact sequence of $G$-modules

$$
E^{G}: 0 \longrightarrow L^{G} \stackrel{\lambda^{\prime}}{\longrightarrow} M^{G} \stackrel{\mu^{\prime}}{\longrightarrow} N^{G} \longrightarrow 0,
$$

where $\lambda^{\prime}=1 \otimes \lambda, \mu^{\prime}=1 \otimes \mu$. One can find examples where $E$ is $H$-split but $E^{G}$ is not. However, we prove $\left({ }^{2}\right)$

(2.1) Proposition. Suppose that $G$ can be written as a disjoint union $G=g_{1} K \cup \cdots$ $\cup g_{s} K$, where the elements $\left\{g_{i}\right\}$ are such that $g_{1} H \cup \cdots \cup g_{s} H=H g_{1} \cup \cdots \cup H g_{s}$. (This is surely possible when $H \triangle G$, for example.) Then for each $H$-split $K$-exact

$\left(^{2}\right)$ This generalizes Lemma 2.6 of [4]. 
sequence $E$, the G-exact sequence $E^{G}$ is also $H$-split. In this case, therefore, the induction map $[M] \rightarrow\left[M^{G}\right]$ defines an additive homomorphism $a(K, H) \rightarrow a(G, H)$.

Proof. We may write

$$
L^{G}=\sum_{i=1}^{s} g_{i} \otimes L, \quad M^{G}=\sum_{i=1}^{s} \oplus g_{i} \otimes M,
$$

and for each $i$,

$$
\lambda^{\prime}\left(g_{i} \otimes l\right)=g_{i} \otimes \lambda(l), \quad l \in L .
$$

Since the sequence $E$ is $H$-split, there exists an $H$-retraction of $\lambda$, that is, there exists a map $\rho \in \operatorname{Hom}_{H}(M, L)$ such that $\rho \lambda=1$ on $L$. Define $\rho^{\prime} \in \operatorname{Hom}_{\Omega}\left(M^{G}, L^{G}\right)$ by

$$
\rho^{\prime}\left(g_{i} \otimes m\right)=g_{i} \otimes \rho(m), \quad m \in M, 1 \leqq i \leqq s .
$$

Then $\rho^{\prime}$ is well defined, and $\rho^{\prime} \lambda^{\prime}=1$ on $L^{G}$. It remains to prove that $\rho^{\prime}$ is an $H$ homomorphism.

Fix the integer $i, 1 \leqq i \leqq r$, and let $x \in H, m \in M$. Since $x g_{i} \in H g_{i}$, the hypothesis implies that $x g_{i}=g_{j} y$ for some $y \in H$ and some $j, 1 \leqq j \leqq r$. Therefore

$$
\begin{aligned}
\rho^{\prime}\left(x \cdot\left(g_{i} \otimes m\right)\right) & =\rho^{\prime}\left(x g_{i} \otimes m\right)=\rho^{\prime}\left(g_{j} \otimes y m\right)=g_{j} \otimes \rho(y m) \\
& =g_{j} \otimes y \rho(m)=x g_{i} \otimes \rho(m)=x \cdot \rho^{\prime}\left(g_{i} \otimes m\right) .
\end{aligned}
$$

Hence $\rho^{\prime}$ is an $H$-homomorphism, and the proposition is established.

Given a $G$-module $M$, if there exists an $H$-split $G$-exact sequence (1.1) with $L \neq 0, N \neq 0$, then of course $[M]=[L]+[N]$ in $a(G, H)$. If no such sequence exists, call $M$ an $H$-simple $G$-module. Thus, $M$ is $H$-simple if and only if no nontrivial $G$-submodule of $M$ is an $H$-direct summand of $M$. Each $H$-simple $G$-module must be $G$-indecomposable. By way of illustration, we note that the 1 -simple $G$-modules are the irreducible modules, and the $G$-simple $G$-modules are the indecomposable modules.

We have defined $i(G, H)$ to be the additive subgroup of $a(G, G)$ generated by the expressions $\gamma_{E}$ given in (1.2), where $E$ ranges over all $H$-split $G$-exact sequences. Let $i_{0}(G, H)$ be the subgroup of $i(G, H)$ generated by those $\gamma_{E}$ in which the sequence $E$ given by (1.1) ranges over all those $H$-split $G$-exact sequences for which the module $L$ is $H$-simple.

(2.2) Proposition. $i_{0}(G, H)=i(G, H)$.

Proof. It suffices to show that $\gamma_{E} \in i_{0}(G, H)$ for every $H$-split $G$-exact sequence $E$ given by (1.1). We use induction on $\operatorname{dim} L$, and remark that the result follows from the definition of $i_{0}(G, H)$ when $L$ is $H$-simple. Suppose then that $L$ is not $H$-simple, so there exists a nontrivial $H$-split $G$-exact sequence

$$
E_{1}: 0 \rightarrow U \rightarrow L \rightarrow L / U \rightarrow 0 .
$$


By composing the $H$-retractions of $M$ onto $L$, and $L$ onto $U$, we get an $H$-retraction $M \rightarrow U$. Thus there is another $H$-split $G$-exact sequence

$$
E_{2}: 0 \rightarrow U \rightarrow M \rightarrow M / U \rightarrow 0 .
$$

Finally, using the $H$-projection $L \rightarrow L / U$, we obtain an $H$-homomorphism $M \rightarrow$ $L / U$, and hence also there is an $H$-split $G$-exact sequence

$$
E_{3}: 0 \rightarrow L / U \rightarrow M / U \rightarrow M / L \rightarrow 0 .
$$

Since $M / L \cong N$, we find readily that in $a(G, G)$ we have

$$
\gamma_{E}=\gamma_{E_{2}}+\gamma_{E_{3}}-\gamma_{E_{1}} \text {. }
$$

But $\operatorname{dim} U<\operatorname{dim} L$ and $\operatorname{dim} L / U<\operatorname{dim} L$, so by the induction hypothesis each $\gamma_{E_{j}} \in i_{0}(G, H), j=1,2,3$. Therefore also $\gamma_{E} \in i_{0}(G, H)$, as desired.

The remaining propositions in this section are probably well known, but since they are not readily available in the literature, we have included their proofs for the convenience of the reader.

(2.3) Proposition. Let $E$ and $F$ be finite-dimensional algebras over the field $\Omega$, and let $\hat{E}=E / \mathrm{rad} E$, where $\operatorname{rad} E$ is the radical of $E$. Define $\hat{F}$ analogously. If $\hat{E} \cong \Omega$, or more generally if $\hat{E}$ is a separable algebra over $\Omega$, then

$$
\frac{E \otimes F}{\operatorname{rad}(E \otimes F)} \cong \hat{E} \otimes \hat{F}
$$

where $\otimes$ means $\otimes_{\Omega}$.

Proof. Since $\hat{E}$ is separable, the algebra $\hat{E} \otimes \hat{F}$ is semisimple (see [2, §71]). There is an algebra epimorphism $\phi: E \otimes F \rightarrow \hat{E} \otimes \hat{F}$, and $\operatorname{ker} \phi=(\operatorname{rad} E) \otimes F$ $+E \otimes(\operatorname{rad} F)$. Clearly $\operatorname{ker} \phi$ is a nilpotent two-sided ideal in $E \otimes F$, and so $\operatorname{ker} \phi \subset \operatorname{rad}(E \otimes F)$. On the other hand, $\phi$ carries $\operatorname{rad}(E \otimes F)$ onto a nilpotent two-sided ideal in the semisimple algebra $\hat{E} \otimes \hat{F}$, and so $\operatorname{rad}(E \otimes F) \subset \operatorname{ker} \phi$. This proves that $\operatorname{rad}(E \otimes F)=\operatorname{ker} \phi$, and establishes the proposition.

We need next some properties of outer tensor products of modules (see $[2, \S 43]$ ). Let $B$ and $K$ be groups, $M$ a $B$-module, $N$ a $K$-module. Make $M \otimes_{\Omega} N$ into a $(B \times K)$-module, called the outer tensor product of $M$ and $N$ and denoted by $M \# N$, by setting

$$
(x, y)(m \otimes n)=x m \otimes y n, \quad x \in B, y \in K .
$$

(2.4) Proposition. Let $G=B \times K$, and let $M_{1}, M_{2}$ be $B$-modules, and $N_{1}, N_{2}$ $K$-modules. There is an $\Omega$-isomorphism

$$
\psi: \operatorname{Hom}_{B}\left(M_{1}, M_{2}\right) \otimes_{\Omega} \operatorname{Hom}_{K}\left(N_{1}, N_{2}\right) \cong \operatorname{Hom}_{G}\left(M_{1} \# N_{1}, M_{2} \# N_{2}\right),
$$

given by $f \otimes g \rightarrow f \# g$. 
Proof. If $f$ and $g$ are operator homomorphisms, so is $f \# g$. Thus $\psi$ carries $\mathrm{Hom}_{B} \otimes \mathrm{Hom}_{K}$ into $\mathrm{Hom}_{G}$. Furthermore, $\psi$ is monic since there is an $\Omega$-isomorphism

$$
\operatorname{Hom}_{\Omega}\left(M_{1}, M_{2}\right) \otimes_{\Omega} \operatorname{Hom}_{\Omega}\left(N_{1}, N_{2}\right) \cong \operatorname{Hom}_{\Omega}\left(M_{1} \# N_{1}, M_{2} \# N_{2}\right) .
$$

It remains to show that $\psi$ is epic.

By (2.5), each $u \in \operatorname{Hom}_{G}\left(M_{1} \# N_{1}, M_{2} \# N_{2}\right)$ is expressible as

$$
u=\sum_{i=1}^{k} f_{i} \# g_{i}, \quad f_{i} \in \operatorname{Hom}_{\Omega}\left(M_{1}, M_{2}\right), g_{i} \in \operatorname{Hom}_{\Omega}\left(N_{1}, N_{2}\right)
$$

and we may assume that the $\left\{f_{i}\right\}$ are linearly independent over $\Omega$. Then

$$
u(m \otimes n)=\sum f_{i}(m) \otimes g_{i}(n), \quad m \in M_{1}, n \in N_{1} .
$$

Since $u(m \otimes y n)=y \cdot u(m \otimes n), y \in K$, we obtain

$$
\sum f_{i}(m) \otimes\left\{g_{i}(y n)-y g_{i}(n)\right\}=0, \quad m \in M_{1}, n \in N_{1}, y \in K .
$$

But the $\left\{f_{i}\right\}$ are linearly independent, so we may choose $m \in M_{1}$ such that $f_{1}(m) \neq 0$, $f_{2}(m)=0, \ldots, f_{k}(m)=0$. This yields $g_{1}(y n)=y g_{1}(n), n \in N_{1}, y \in K$, so that $g_{1} \in \operatorname{Hom}_{K}\left(N_{1}, N_{2}\right)$. The same holds for $g_{2}, \ldots, g_{k}$, and we may thus rewrite $u$ as

$$
u=\sum f_{j}^{\prime} \# g_{j}^{\prime}, \quad f_{j}^{\prime} \in \operatorname{Hom}_{\Omega}\left(M_{1}, M_{2}\right), g_{j}^{\prime} \in \operatorname{Hom}_{K}\left(N_{1}, N_{2}\right),
$$

where now the $\left\{g_{j}^{\prime}\right\}$ are linearly independent over $\Omega$. The preceding argument can be used again, so that each $f_{j}^{\prime} \in \operatorname{Hom}_{B}\left(M_{1}, M_{2}\right)$. This proves that $u \in$ image of $\psi$, as desired.

For a $B$-module $M$, we put $E(M)=\operatorname{Hom}_{B}(M, M), \hat{E}(M)=E(M) / \operatorname{rad} E(M)$, and we use analogous notation for other modules.

(2.6) Proposition. Let $M$ be a B-module, $N$ a $K$-module. Then $E(M \# N) \cong$ $E(M) \otimes E(N)$, where $\otimes$ is $\otimes_{\Omega}$. If $M$ and $N$ are indecomposable, and if $\hat{E}(M) \cong \Omega$, then $M \# N$ is also indecomposable. In particular, $M \# N$ is indecomposable if $M$ is irreducible, $N$ indecomposable, and $\Omega$ is a splitting field for the group $B$.

Proof. The first assertion follows at once from (2.4). Next, a module $M$ is indecomposable if and only if $E(M)$ is completely primary (see [2, §54]), that is, if and only if $\hat{E}(M)$ is a skewfield. Suppose now that $M$ and $N$ are indecomposable, and that $\hat{E}(M) \cong \Omega$. By $(2.3)$,

$$
\hat{E}(M \# N) \cong \frac{E(M) \otimes E(N)}{\operatorname{rad}(E(M) \otimes E(N))} \cong \hat{E}(M) \otimes \hat{E}(N) \cong \hat{E}(N)
$$

But $\hat{E}(N)$ is a skewfield, whence so is $\hat{E}(M \# N)$, and thus $M \# N$ is indecomposable.

If $M$ is irreducible, and $\Omega$ is a splitting field for $B$, then $E(M) \cong \Omega$, and so also $\hat{E}(M) \cong \Omega$. This completes the proof. 
3. Excision of normal $p$-subgroups. We begin with a simple result which shows how one may obtain relations in a relative Grothendieck ring. (A special case of this lemma was used in [4, Lemma 3.5].)

(3.1) Pushout Lemma. Let $M, U, V$ be $G$-modules, and let $\alpha: M \rightarrow U, \beta: M \rightarrow V$ be G-monomorphisms. Suppose that there exist H-homomorphisms $\theta: U \rightarrow V$, $\eta: V \rightarrow U$ making the following diagram commute:

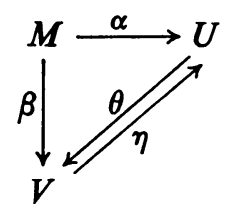

Then in $a(G, H)$ there is a relation $[V]-[V / \beta M]=[U]-[U / \alpha M]$.

Proof. Define the $G$-module $X$ by the pushout diagram

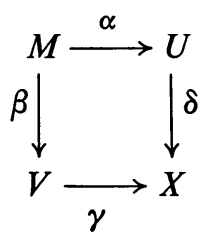

so that

$$
X=(U \oplus V) /\{(\alpha m,-\beta m): m \in M\} .
$$

Then $\gamma$ and $\delta$ are $G$-monomorphisms, and $U / \alpha M \cong X / \gamma V$. Now the $G$-exact sequence $0 \rightarrow V \stackrel{\gamma}{\rightarrow} X \rightarrow X / \gamma V \rightarrow 0$ is $H$-split if and only if there exists a map $\theta \in \operatorname{Hom}_{H}(U, V)$ such that $\theta \alpha=\beta$. Since such a map $\theta$ exists by hypothesis, this sequence is $H$-split, and so in $a(G, H)$ we have the formula

$$
[X]=[V]+[X / \gamma V]=[V]+[U / \alpha M] \text {. }
$$

Similarly,

$$
[X]=[U]+[V / \beta M] .
$$

Eliminating $[X]$ between these two equations gives the desired formula in $a(G, H)$.

(3.2) THEOREM. (Excision of normal p-subgroups.) Let $G$ be a semidirect product $P \cdot K$, where $P \triangle G, P \cap K=1$, and $P$ is a p-group. Let $H \subset K$. Then the restriction map $\varphi: a(G, H) \rightarrow a(K, H)$ is a ring isomorphism.

Proof. Each $K$-module $M$ determines a $G$-module $M^{\prime}$, consisting of the same elements as $M$, with the action of $G$ defined by using the homomorphism $G \rightarrow G / P$ $\cong K$. In other words, the $K$-module $M$ becomes the $G$-module $M^{\prime}$ on which the elements of $P$ act trivially. The map $\psi: a(K, H) \rightarrow a(G, H)$, given by $[M] \rightarrow\left[M^{\prime}\right]$, 
is thus a well-defined ring homomorphism. If the subscript $K$ denotes restriction to $K$, then for each $K$-module $M$ we have

$$
\varphi \psi[M]=\varphi\left[M^{\prime}\right]=\left[\left(M^{\prime}\right)_{K}\right]=[M] .
$$

Thus $\varphi \psi=1$, so in order to prove that $\varphi$ is a ring isomorphism with inverse $\psi$, it suffices to show that $\psi$ is an epimorphism.

Let $L$ be a $G$-module, and consider the element $[L] \in a(G, H)$. We shall show by induction on $\operatorname{dim} L$ that $[L] \in$ image of $\psi$ always. Let $M$ be an irreducible $G$ submodule of $L$. Since $P$ is a normal $p$-subgroup of $G, P$ acts trivially on $M$; therefore the mapping $\alpha: M \rightarrow\left(L_{K}\right)^{\prime}$ is a $G$-monomorphism. Let $\beta: M \rightarrow L$ be the inclusion map. In order to use the Pushout Lemma, we must find $H$-homomorphisms $\theta, \eta$ making the following diagram commute:

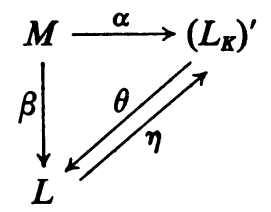

Since $H \subset K$, and $\left(L_{K}\right)^{\prime}$ is the lifting of $L_{K}$ to a $G$-module with trivial action of $P$, we may in fact choose $\theta$ and $\eta$ to be the identity map on $L$.

It follows now from (3.1) that in $a(G, H)$ we have the equality

$$
[L]=\left[\left(L_{K}\right)^{\prime}\right]-\left[\left(L_{K}\right)^{\prime} / \alpha M\right]+[L / \beta M] .
$$

The first two terms on the right lie in the image of $\psi$, from the definition of $\psi$. The last term also lies in the image of $\psi$ by the induction hypothesis, since $\operatorname{dim}(L / \beta M)<\operatorname{dim} L$. This completes the proof.

Keeping the notation used in the above proof, we note that $\varphi$ and $\psi$ have been shown to be inverses of one another. Therefore also $\psi \varphi=1$, which means that for each $G$-module $L$, there is an equality in $a(G, H):[L]=\left[\left(L_{K}\right)^{\prime}\right]$.

We list also the following simple consequence of the theorem.

(3.3) Corollary. Let $P$ be any p-group. Then the restriction map a $(P \times H, H)$ $\rightarrow a(H, H)$ is a ring isomorphism of the relative Grothendieck ring $a(P \times H, H)$ onto the Green ring $a(H, H)$.

Next we give an example to show how the Excision Theorem may be used to calculate $a(G, H)$. Take $\Omega$ any field of characteristic 2, and let $G=S_{4}$ (symmetric group on the symbols $1,2,3,4), K=$ subgroup of $G$ consisting of the permutations on the symbols $1,2,3$, and $H=\{(1),(12)\}$. If we choose

$$
P=\{(1),(12)(34),(13)(24),(14)(23)\},
$$

then $P \triangle G$, and $G$ is a semidirect product $P \cdot K$. The theorem then implies that $a(G, H) \cong a(K, H)$, the isomorphism being given by restriction from $G$ to $K$. 
However, $H$ is a Sylow 2-subgroup of $K$, so that an exact sequence of $K$-modules is $H$-split if and only if it is $K$-split (see [2, §63]). Therefore $a(K, H)=a(K, K)$, and the latter is just the Green ring of $S_{3}$. Since the indecomposable $S_{3}$-modules are easily determined, the structure of $a(G, H)$ is completely known in this case.

We conclude the section with a somewhat deeper consequence of the Excision Theorem. Recall that a $G$-module $M$ is called $(G, H)$-projective if $M$ is a $G$-direct summand of an induced module $X^{G}$, for some $H$-module $X$. These $(G, H)$ projectives generate an additive subgroup $k(G, H)$ of the Green ring $a(G, G)$, and indeed $k(G, H)$ is an ideal in $a(G, G)$. Furthermore, it is easily found that

$$
k(G, H) \cdot i(G, H)=0 .
$$

There is a ring homomorphism

$$
\kappa: k(G, H) \rightarrow a(G, H)
$$

defined by composition of maps:

$$
k(G, H) \rightarrow a(G, G) \rightarrow a(G, G) / i(G, H)=a(G, H),
$$

where the first map is inclusion. We have called $\kappa$ the Cartan homomorphism for the pair $(G, H)$, since in the special case where $H=1$, the matrix of $\kappa$ is the Cartan matrix associated with the group algebra $\Omega G$.

Making use of a result of Conlon [1], we now prove that $\kappa$ is monic. This fact was established in [4] under the restrictive hypothesis that $H \triangle G$. As we shall see, this restriction is unnecessary. Let $C$ be the complex field, and set

$$
K(G, H)=C \otimes_{z} k(G, H), \quad A(G, H)=C \otimes_{z} a(G, H),
$$

and define $A(G, G), I(G, H)$ analogously. There is a commutative diagram

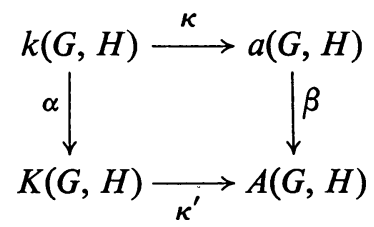

where $\alpha, \beta$ arise from tensoring with $C$, and where $\kappa^{\prime}$ is defined by composition of maps

$$
K(G, H) \rightarrow A(G, G) \rightarrow A(G, G) / I(G, H) \cong A(G, H) .
$$

It follows at once that

$$
\operatorname{ker} \kappa^{\prime}=K(G, H) \cap I(G, H),
$$

while from (3.4) we have

$$
K(G, H) \cdot I(G, H)=0 .
$$


Now $k(G, H)$ is $Z$-free, since it is a $Z$-submodule of the free $Z$-module $a(G, G)$. Therefore $\alpha$ is monic. To prove $\kappa$ monic, it thus suffices to show $\kappa^{\prime}$ monic. By Conlon [1, Theorem 3.21(a)], $K(G, H)$ is an ideal direct summand of $A(G, G)$. Hence there exists an idempotent $e \in A(G, G)$ such that $K(G, H)=e \cdot A(G, G)$. By (3.6) we deduce that $e \cdot I(G, H)=0$. However, $e$ acts as the identity on $K(G, H)$, and therefore $K(G, H) \cap I(G, H)=0$. This completes the proof that $\kappa^{\prime}$ is monic, and thus also that $\kappa$ is monic.

We restate our conclusion as follows.

(3.7) Proposition. The Cartan homomorphism $\kappa: k(G, H) \rightarrow a(G, H)$ is monic. Thus, if $M$ and $N$ are $(G, H)$-projective $G$-modules, then $M \cong N$ if and only if $[M]=[N]$ in $a(G, H)$.

From this we may deduce

(3.8) Theorem. Let $G=P \cdot K, P \triangle G, P=p$-group, $P \cap K=1$. Let $M$ and $N$ be $(G, K)$-projective $G$-modules. Then $M$ and $N$ are $G$-isomorphic if and only if they are K-isomorphic.

Proof. By (3.7), $M \cong N$ if and only if $[M]=[N]$ in $a(G, K)$. From the Excision Theorem 3.2 with $H=K$, it follows that the restriction map gives an isomorphism $a(G, K) \cong a(K, K)$. Thus $[M]=[N]$ in $a(G, K)$ if and only if $\left[M_{K}\right]=\left[N_{K}\right]$ in $a(K, K)$. The latter equality holds if and only if $M_{K} \cong N_{K}$, since $a(K, K)$ is the Green ring of $K$. This completes the proof.

It would be of interest to give a direct proof of the preceding theorem.

4. Direct product with $p$-free factor. We begin with an easy result.

(4.1) Proposition. Let $G=B \times K$ be a direct product of groups, and let $H \subset K$. Define

$$
\tau: a(B, 1) \otimes_{z} a(K, H) \rightarrow a(G, H)
$$

by letting $[X] \otimes[M] \rightarrow[X \# M]$, where $X \# M$ is the outer tensor product of modules (see §2). Then $\tau$ is a well-defined ring homomorphism.

Proof. In order to prove that $\tau$ is well defined, we must verify two things:

(i) If $0 \rightarrow X_{1} \rightarrow X_{2} \rightarrow X_{3} \rightarrow 0$ is an exact sequence of $B$-modules, then for each $K$-module $M$, the sequence

$$
0 \rightarrow X_{1} \# M \rightarrow X_{2} \# M \rightarrow X_{3} \# M \rightarrow 0
$$

is an $H$-split $G$-exact sequence. This is indeed the case; exactness is clear, and the sequence is $H$-split since $\left(X_{i} \# M\right)_{H}$ is a direct sum of $\left(X_{i}: \Omega\right)$ copies of $M_{H}$.

(ii) If $0 \rightarrow L \rightarrow M \rightarrow N \rightarrow 0$ is an $H$-split $K$-exact sequence, then for each $B$ module $X$, the sequence

$$
0 \rightarrow X \# L \rightarrow X \# M \rightarrow X \# N \rightarrow 0
$$


is $H$-split and $G$-exact. Again, exactness is clear; further, if $\mu: M \rightarrow L$ is an $H$ retraction splitting the original $K$-exact sequence, then $1 \# \mu: X \# M \rightarrow X \# L$ is an $H$-retraction splitting the $G$-exact sequence.

This proves that $\tau$ is a well-defined additive homomorphism, since we extend it by linearity. But $\tau$ is also a ring homomorphism, by virtue of the $G$-isomorphism

$$
(X \# M) \otimes\left(X^{\prime} \# M^{\prime}\right) \cong\left(X \otimes X^{\prime}\right) \#\left(M \otimes M^{\prime}\right),
$$

where $\otimes$ means $\otimes_{\Omega}$.

For the remainder of this section, let $G=A \times K$, where $H \subset K$, and where $A$ is a $p$-free group, that is, $p$ does not divide $[A: 1]$. Suppose also that $\Omega$ is a splitting field for $A$. Since $K$ contains a Sylow $p$-subgroup of $G$, it follows that every $G$ module is $(G, K)$-projective, and hence that every indecomposable $G$-module $L$ is a $G$-direct summand of an induced module $M^{G}$ for some indecomposable $K$ module $M$ (see [2, §63]). However,

$$
M^{G}=\Omega G \otimes_{\Omega K} M \cong \Omega A \# M \cong \sum^{\oplus}\left(X_{i} \# M\right),
$$

where the $\left\{X_{i}\right\}$ are irreducible $A$-modules such that $\Omega A=\Sigma^{\oplus} X_{i}$. By the last statement in (2.6), each $X_{i} \# M$ is indecomposable; hence $L$ must be isomorphic to some $X_{i} \# M$, and therefore $[L]$ lies in the image of $\tau$. Since every $G$-module is a direct sum of indecomposables, this proves that the map

$$
\tau: a(A, 1) \otimes_{\mathrm{z}} a(K, H) \rightarrow a(G, H)
$$

is a surjective ring homomorphism in this case.

Consider for a moment the special case where $H=K$. Since $K$ contains a Sylow $p$-subgroup of $G$, a $G$-exact sequence is $K$-split if and only if it is $G$-split. Hence the relative Grothendieck ring $a(G, K)$ coincides with the Green ring $a(G, G)$ in this case, and as in (4.2) there is a surjective ring homomorphism

$$
\tau_{0}: a(A, 1) \otimes_{\mathrm{z}} a(K, K) \rightarrow a(G, G) .
$$

Now let $X$ range over the distinct irreducible $A$-modules, and $M$ over the distinct indecomposable $K$-modules. Then the products $[X] \otimes[M]$ form a free $Z$-basis for $a(A, 1) \otimes a(K, K)$, and

$$
\tau_{0}:[X] \otimes[M] \rightarrow[X \# M] .
$$

If $X \# M \cong X^{\prime} \# M^{\prime}$, with $X, X^{\prime}$ irreducible and $M, M^{\prime}$ indecomposable, then by (2.4) we may conclude that $\operatorname{Hom}_{A}\left(X, X^{\prime}\right) \neq 0$, and hence that $X \cong X^{\prime}$. Furthermore, $(X \# M)_{K}$ is a direct sum of $(X: \Omega)$ copies of $M$, whence also $M \cong M^{\prime}$. Thus the elements $[X \# M]$ form a free $Z$-basis for the Green ring $a(G, G)$, which proves that the map $\tau_{0}$ in (4.3) is a ring isomorphism.

We shall use this fact in proving

(4.4) Theorem. Let $G=A \times K$, where $H \subset K$ and where $A$ is a p-free group. Assume that $\Omega$ is a splitting field for $A$. Then the map $\tau$ given by (4.2) is a ring isomorphism. 
Proof. We have already shown that $\tau$ is a ring epimorphism, and it remains to prove that $\tau$ is monic. It follows from the definition of relative Grothendieck rings that there are two exact sequences of additive groups:

$$
\begin{aligned}
& 0 \rightarrow i(K, H) \rightarrow a(K, K) \rightarrow a(K, H) \rightarrow 0, \\
& 0 \rightarrow i(G, H) \rightarrow a(G, G) \rightarrow a(G, H) \rightarrow 0 .
\end{aligned}
$$

We apply $a(A, 1) \otimes_{z} \cdot$ to the first sequence; this preserves exactness since $a(A, 1)$ is $Z$-free. Therefore we obtain a commutative diagram of $Z$-modules, with exact rows:

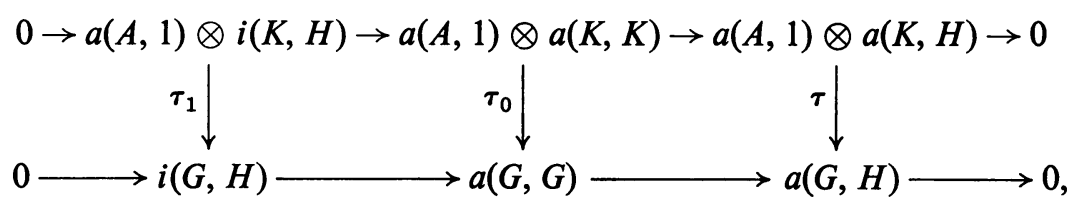

where $\otimes$ is $\otimes_{z}$, and each $\tau$ is given by \#. The image of $\tau_{1}$ clearly lies in $i(G, H)$, and we have already shown that $\tau_{0}$ is an isomorphism. To complete the proof, it thus suffices to show that $\tau_{1}$ is epic.

Consider any $H$-split $G$-exact sequence

$$
E: 0 \longrightarrow L \stackrel{\lambda}{\longrightarrow} M \stackrel{\mu}{\longrightarrow} N \longrightarrow 0
$$

in which $L$ is $H$-simple, and set $\gamma_{E}=[M]-[L]-[N] \in a(G, G)$. By Proposition 2.2, such $\gamma_{E}$ 's generate $i(G, H)$, and so we need only show that $\gamma_{E} \in$ image of $\tau_{1}$. Since $L$ is an $H$-simple $G$-module, it must be $G$-indecomposable, and so we may write $L=X \# T$ for some irreducible $A$-module $X$ and some indecomposable $K$-module $T$. Let us express $M$ as a direct sum of indecomposable $G$-modules, say $M=$ $\Sigma^{\oplus} X_{i} \# T_{i}, X_{i}$ irreducible, $T_{i}$ indecomposable. Collecting terms with common first factor, we may then write $M=\Sigma^{\oplus} X_{i} \# U_{i}$, where now the $X_{i}$ are distinct irreducible $A$-modules, and the $K$-modules $U_{i}$ may be decomposable. However, there exists a nonzero $\lambda \in \operatorname{Hom}_{G}(L, M)$, defined in the sequence $E$, and so by Proposition 2.4 some one of the $X_{i}$ 's must be isomorphic to $X$. To fix the notation, suppose that $X_{1} \cong X$; by (2.4), we then have $\lambda(L) \subset X_{1} \# U_{1}$. Replacing $L$ by a module isomorphic to it, we may assume that $X$ coincides with $X_{1}$, so now we have

$$
L=X_{1} \# T, \quad M=X_{1} \# U_{1} \oplus \sum_{i>1}^{\oplus} X_{i} \# U_{i}
$$

and $\lambda: X_{1} \# T \rightarrow X_{1} \# U_{1}$ is a $G$-monomorphism. However, $\operatorname{Hom}_{A}\left(X_{1}, X_{1}\right) \cong \Omega$ since $\Omega$ is a splitting field for $A$, and so by (2.4) we may write $\lambda=1 \# g$ for some monomorphism $g \in \operatorname{Hom}_{K}\left(T, U_{1}\right)$. Thus there is a $G$-exact sequence

$$
E_{1}: 0 \longrightarrow X_{1} \# T_{1} \stackrel{1 \# g}{\longrightarrow} X_{1} \# U_{1} \longrightarrow X_{1} \# \text { coker } g \longrightarrow 0 \text {. }
$$


Now let $\rho \in \operatorname{Hom}_{H}(M, L)$ be an $H$-retraction which splits $E$, so that $\rho \lambda=1$ on $L$; let $\rho_{1}$ be the restriction of $\rho$ to the summand $X_{1} \# U_{1}$ of $M$. Since $\lambda(L) \subset X_{1} \# U_{1}$, we see that $\rho_{1} \lambda=1$ on $L$, and thus the sequence $E_{1}$ is $H$-split. But then the sequence $E_{1}$ is also $(A \times H)$-split, since $H$ contains a Sylow $p$-subgroup of $A \times H$. Hence there exists a map

$$
\theta \in \operatorname{Hom}_{A \times H}\left(X_{1} \# U_{1}, X_{1} \# T\right)
$$

such that $\theta(1 \# g)=1$. By (2.4) we may write $\theta$ in the form $\theta=1 \# f$ for some $f \in \operatorname{Hom}_{H}\left(U_{1}, T\right)$; in that case it follows that $f g=1$ on $T$. Therefore the sequence

$$
E_{2}: 0 \longrightarrow T \stackrel{g}{\longrightarrow} U_{1} \longrightarrow \text { coker } g \longrightarrow 0
$$

is an $H$-split $K$-exact sequence. Consequently

$$
\gamma_{E_{1}}=\tau_{1}\left\{\left[X_{1}\right] \otimes \gamma_{E_{2}}\right\} \in \text { image of } \tau_{1} .
$$

We are trying to prove that $\gamma_{E}$ lies in the image of $\tau_{1}$. However, in $a(G, G)$ we have the equations

$$
\begin{aligned}
\gamma_{E} & =[M]-[L]-[M / L] \\
& =\left\{\left[X_{1} \# U_{1}\right]+\sum_{i>1}\left[X_{i} \# U_{i}\right]\right\}-\left[X_{1} \# T\right]-\left\{\left[X_{1} \# \text { coker } g\right]+\sum_{i>1}\left[X_{i} \# U_{i}\right]\right\} \\
& =\gamma_{E_{1}} .
\end{aligned}
$$

This completes the proof of the theorem.

5. Direct product in general case. The main result of this article is as follows:

(5.1) TheOReM. Let $G$ be a direct product $B \times K$ of arbitrary finite groups $B$ and $K$, and let $H \subset K$. Assume that the field $\Omega$ is a splitting field for $B$ and all of its subgroups. Then the map

$$
\tau: a(B, 1) \otimes_{z} a(K, H) \rightarrow a(G, H)
$$

defined by $[X] \otimes[M] \rightarrow[X \# M]$, is a ring isomorphism.

We have already shown in (4.1) that $\tau$ is a ring homomorphism. Furthermore, we have established the above theorem for the special case where the first factor $B$ is $p$-free (see (4.4)). An easy argument, based on the material in $\$ 3$, shows that the theorem is also valid when $B$ is a $p$-group; however, we shall not use the results of $\S 3$ in this form, but will rely directly on Theorem 3.2 .

The proof of Theorem 5.1 for the general case depends on reducing it to the two special cases mentioned above, the key device being the Brauer Induction Theorem (see [2, \$40]). The quickest way of accomplishing this reduction is by means of Lam's theory of Frobenius functors [3]. In order to make this article self-contained, however, we have included a direct proof of the reduction step. 
Let us begin the proof of Theorem 5.1 with a simple observation. Let $B_{1}$ be a subgroup of $B$, and set $G_{1}=B_{1} \times K$; remember that $G=B \times K$, and that $H \subset K$. There are restriction maps

$$
r: a(B, 1) \rightarrow a\left(B_{1}, 1\right), \quad r^{\prime}: a(G, H) \rightarrow a\left(G_{1}, H\right) .
$$

On the other hand, if $B=\bigcup b_{i} B_{1}$ is a coset decomposition of $B$, then $G=\bigcup b_{i} G_{1}$ is a coset decomposition of $G$. Clearly $\bigcup b_{i} H=\bigcup H b_{i}$, so by (2.1) there exists an induction map $i^{\prime}: a\left(G_{1}, H\right) \rightarrow a(G, H)$, where $i^{\prime}[M]=\left[M^{G}\right], \quad M=G_{1}$-module. Finally, there is an induction map $i: a\left(B_{1}, 1\right) \rightarrow a(B, 1)$, given by $i:[U] \rightarrow\left[U^{B}\right]$, $U=B_{1}$-module. The induction and restriction maps are connected by the usual Frobenius relations (see [5], for example):

$$
\begin{gathered}
u \cdot i(v)=i(r(u) \cdot v), \quad u \in a(B, 1), v \in a\left(B_{1}, 1\right), \\
x \cdot i^{\prime}(y)=i^{\prime}\left(r^{\prime}(x) \cdot y\right), \quad x \in a(G, H), y \in a\left(G_{1}, H\right) .
\end{gathered}
$$

Next we note that there is a diagram consisting of two commutative squares:

$$
\begin{array}{cl}
a(B, 1) \otimes_{z} a(K, H) \stackrel{\tau}{\longrightarrow} a(G, H) \\
r \otimes 1|| i \otimes 1 & r^{\prime} \downarrow \mid i^{\prime} \\
a\left(B_{1}, 1\right) \otimes_{z} a(K, H) \underset{\tau_{1}}{\longrightarrow} a\left(G_{1}, H\right)
\end{array}
$$

that is,

$$
\tau_{1}(r \otimes 1)=r^{\prime} \tau, \quad \tau(i \otimes 1)=i^{\prime} \tau_{1} .
$$

We apply this first to the case where $B=A \times P, B_{1}=A$, with $A$ a $p$-free group and $P$ a $p$-group. There is a commutative diagram

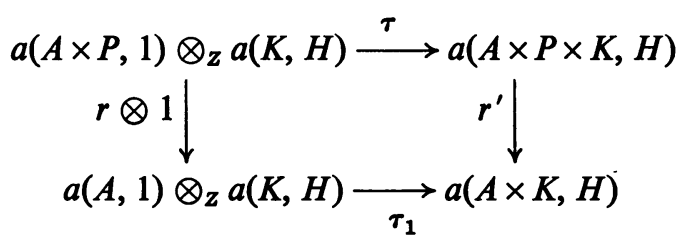

Now the map $r: a(A \times P, 1) \rightarrow a(A, 1)$ is an isomorphism, since $P$ acts trivially on every irreducible $(A \times P)$-module. Thus $r \otimes 1$ is also an isomorphism. Theorem 3.2 tells us that $r^{\prime}$ is an isomorphism, while $\tau_{1}$ is an isomorphism by Theorem 4.4. This implies that the map $\tau$ in (5.5) is an isomorphism, that is,

$$
a(A \times P, 1) \otimes_{z} a(K, H) \cong a(A \times P \times K, H) .
$$

Let $1_{B}$ be the 1-representation of $B$. From the Brauer Induction Theorem [2, $\S 40]$ it follows that there exist elementary subgroups $B_{\alpha}$ of $B$, and elements 
$x_{\alpha} \in a\left(B_{\alpha}, 1\right)$, such that

$$
\left[1_{B}\right]=\sum_{\alpha} i_{\alpha}\left(x_{\alpha}\right) \text { in } a(B, 1)
$$

where $i_{\alpha}: a\left(B_{\alpha}, 1\right) \rightarrow a(B, 1)$ is the induction map. (Actually, the theorem asserts such a result for representations over a splitting field of characteristic zero, but this easily implies the same result for modular representations.) Each elementary subgroup $B_{\alpha}$ can be written as a direct product $A_{\alpha} \times P_{\alpha}$, with $A_{\alpha} p$-free and $P_{\alpha}$ a $p$-group. By (5.6) we know that

$$
\tau_{\alpha}: a\left(B_{\alpha}, 1\right) \otimes_{z} a(K, H) \rightarrow a\left(B_{\alpha} \times K, H\right)
$$

is a ring isomorphism, where $\tau_{\alpha}$ is given by outer tensor product.

For each $\alpha$, there is a diagram consisting of two commutative squares:

$$
\begin{array}{cc}
a(B, 1) \otimes_{z} a(K, H) \stackrel{\tau}{\longrightarrow} a(B \times K, H) \\
r_{\alpha} \otimes 1 \mid \uparrow_{i_{\alpha}} \otimes 1 & r_{\alpha}^{\prime} \downarrow \uparrow i_{\alpha}^{\prime} \\
a\left(B_{\alpha}, 1\right) \otimes_{z} a(K, H) \underset{\tau_{\alpha}}{\longrightarrow} a\left(B_{\alpha} \times K, H\right)
\end{array}
$$

As remarked previously, the map $\tau$ defined in the statement of Theorem 5.1 is a ring homomorphism. Let us show now that $\tau$ is monic. Suppose that $\tau(v)=0$, where

$$
v=\sum_{j} u_{j} \otimes t_{j}, \quad u_{j} \in a(B, 1), t_{j} \in a(K, H) .
$$

Then for each $\alpha, 0=r_{\alpha}^{\prime} \tau(v)=\tau_{\alpha}\left(r_{\alpha} \otimes 1\right) v$. Since $\tau_{\alpha}$ is monic, this gives $\left(r_{\alpha} \otimes 1\right) v=0$, that is,

$$
\sum_{j} r_{\alpha}\left(u_{j}\right) \otimes t_{j}=0
$$

Using (5.7), (5.2) and (5.9), we obtain

$$
\begin{aligned}
v & =v\left\{\left[1_{B}\right] \otimes\left[1_{E}\right]\right\}=\left(\sum_{j} u_{j} \otimes t_{j}\right)\left(\sum i_{\alpha}\left(x_{\alpha}\right) \otimes\left[1_{K}\right]\right) \\
& =\sum_{j, \alpha}\left(u_{j} \cdot i_{\alpha}\left(x_{\alpha}\right)\right) \otimes t_{j}=\sum_{j, \alpha} i_{\alpha}\left(r_{\alpha}\left(u_{j}\right) \cdot x_{\alpha}\right) \otimes t_{j} \\
& =\sum_{\alpha}\left(\left(i_{\alpha} \otimes 1\right) \cdot\left\{\left(\sum_{j} r_{\alpha}\left(u_{j}\right) \otimes t_{j}\right) \cdot\left(x_{\alpha} \otimes 1\right)\right\}\right)=0 .
\end{aligned}
$$

This completes the proof that $\tau$ is monic.

In order to show that $\tau$ is epic, we note first that by (5.7),

$$
\begin{aligned}
{\left[1_{G}\right] } & =\tau\left\{\left[1_{B}\right] \otimes\left[1_{K}\right]\right\}=\tau\left\{\sum_{\alpha} i_{\alpha}\left(x_{\alpha}\right) \otimes\left[1_{K}\right]\right\} \\
& =\tau \sum_{\alpha}\left(i_{\alpha} \otimes 1\right)\left(x_{\alpha} \otimes\left[1_{K}\right]\right)=\sum_{\alpha} i_{\alpha}^{\prime} \tau_{\alpha}\left(y_{\alpha}\right),
\end{aligned}
$$


where $y_{\alpha}=x_{\alpha} \otimes\left[1_{K}\right]$. Therefore for $u \in a(B \times K, H)$ we have (by (5.3))

$$
\begin{aligned}
u & =u \cdot\left[1_{G}\right]=u \cdot \sum_{\alpha} i_{\alpha}^{\prime} \tau_{\alpha}\left(y_{\alpha}\right) \\
& =\sum_{\alpha} i_{\alpha}^{\prime}\left\{r_{\alpha}^{\prime}(u) \cdot \tau_{\alpha}\left(y_{\alpha}\right)\right\} .
\end{aligned}
$$

But for each $\alpha$ the map $\tau_{\alpha}$ is epic, and so there exists an element

$$
z_{\alpha} \in a\left(B_{\alpha}, 1\right) \otimes a(K, H)
$$

such that $r_{\alpha}^{\prime}(u) \cdot \tau_{\alpha}\left(y_{\alpha}\right)=\tau_{\alpha}\left(z_{\alpha}\right)$. Hence

$$
u=\sum_{\alpha} i_{\alpha}^{\prime} \tau_{\alpha}\left(z_{\alpha}\right)=\tau\left\{\sum_{\alpha}\left(i_{\alpha} \otimes 1\right) z_{\alpha}\right\}
$$

that is, $u$ lies in the image of $\tau$. This completes the proof of the theorem.

\section{REFERENCES}

1. S. B. Conlon, Relative components of representations, J. Algebra 8 (1968), 478-501.

2. C. W. Curtis and I. Reiner, Representation theory of finite groups and associative algebras, Pure and Applied Mathamatics, Vol. 11, Interscience, New York, 1962.

3. T. Y. Lam, Induction theorems for Grothendieck groups and Whitehead groups of finite groups, Ann. Sci. École Norm. Sup. (4) 1 (1968), 91-148.

4. T. Y. Lam and I. Reiner, Relative Grothendieck groups, J. Algebra 11 (1969), 213-242.

5. R. G. Swan, Induced representations and projective modules, Ann. of Math. (2) 71 (1960), 552-578.

\section{UNIVERSITY of CALIFORNia, \\ Berkeley, California \\ UNIVERSITY OF ILLINOIS, \\ URBANA, ILLINOIS}

